
\title{
25 Research Soure \\ Is Maternal Hypertension a Reducing Factor for Intraventricular Hemorrhage in Iranian Preterm Newborns?
}

\section{Mandana Kashaki}

Shahid Akbarabadi Clinical Research Development Unit (ShACRDU), Iran University of Medical Sciences (IUMS)

\section{Maryam Saboute}

Shahid Akbarabadi Clinical Research Development Unit (ShACRDU), Iran University of Medical Sciences

Maryam Esmaeili Allafi

Iran University of Medical Sciences (IUMS)

Elahe Norouzi ( $\nabla$ elahenorouzimd@gmail.com )

Iran University of Medical Sciences

Research article

Keywords: Preeclampsia, Maternal hypertension, Intraventricular hemorrhage, Prematurity, newborn

Posted Date: November 13th, 2020

DOl: https://doi.org/10.21203/rs.3.rs-105166/v1

License: (c) (i) This work is licensed under a Creative Commons Attribution 4.0 International License. Read Full License 


\section{Abstract}

Background \& objective: Hypertensive disorders of pregnancy including preeclampsia can be associated with preterm birth and infant mortality and morbidity. It seems that intraventricular hemorrhage (IVH) in preterm neonates born to hypertensive mothers is less common compared to the other ones. In this study, we assessed the relationship between hypertensive disorders of pregnant mothers and the incidence of intraventricular hemorrhage in preterm neonates weighing less than 1500 grams.

Methods: In this case-control study, 305 singleton preterm neonates with a birth weight less than $1500 \mathrm{~g}$ were entered. The presence of IVH in all preterm neonates was assessed using brain ultrasonography and its correlation to hypertensive disorders of their mothers were evaluated.

Results: The prevalence of preeclampsia in mothers with and without prepregnancy hypertension was $40 \%$, and $15.09 \%$ respectively. The prevalence of IVH in preterm neonates was $26.9 \%(n=82)$. The incidence of IVH in preterm neonates of mothers with and without preeclampsia was $16.07 \%$, and $29.31 \%$ in $(p=0.028)$, respectively. The prevalence of IVH in preterm neonates of mothers with hypertension and without hypertension was $12.5 \%$ and $29.05 \%$, respectively $(p=0.018)$. Preterm neonates with IVH had a lower mean birth weight, smaller gestational age and lower Apgar scores compared to preterm neonates without IVH with $p$ values of $<0.001,<0.001$ and $<0.01$, respectively.

Conclusion: Hypertensive disorders of pregnancy increases the risk of preterm delivery, but incidence of IVH in preterm neonates weighing less than $1500 \mathrm{gr}$ is lower in infants born to mothers with hypertension including preeclampsia compared to non hypertensive mothers. And also lower gestational age, birth weight, and Apgar scores were important predicting factors for IVH in preterm neonates.

\section{Background}

Intraventricular hemorrhage (IVH) is one of the most common complication and life-threatening events which is associated with high morbidity and mortality in premature infants [1]. Since the blood vessels of the brain in preterm neonates are primitive immature ones, they are very fragile and may easily rupture and cause severe damages to the brain [1,2]. The incidence of IVH in premature infants weighting less than $1500 \mathrm{~g}$ is approximately $25 \%$ [3], but in extremely preterm neonates weighting 500 to $750 \mathrm{~g}$ it occurs in nearly $45 \%$ of infants [4]. IVH can be associated with adverse neurologic sequelae in premature infants, including cerebral palsy, mental retardation, seizures, behavioral or cognitive disabilities $[5,6]$. Some studies reported that premature infants with IVH have significantly lower survival rate compared to those without IVH $[2,7]$. Therefore, identification of predicting factors for IVH is very important for the diagnosis and treatment of the disease. Several factors including genetic and thrombophilic disorders, intrapartum factors, premature infants with respiratory distress syndrome (RDS), cardiovascular factors such as fluctuations in blood pressure are considered as the possible risk factors for IVH [8-10]. Some studies demonstrated that very low birth weight infants, lower gestational age, low Apgar scores, long-term mechanical ventilation, pneumothorax, type of delivery, presence of seizures, thrombocytopenia, RDS and 
infection are important predicting risk factors for IVH in preterm neonates; however, debatable results are available $[1,9,10]$.

Hypertensive disorders of pregnancy including preeclampsia are among the leading causes of maternal mortality and morbidity and subsequently premature births and perinatal morbidity and mortality [11]. Several lines of studies considered preeclampsia as a preventive factor for IVH, but the exact mechanism remains unclear. It may be partly related to magnesium sulfate treatment in mothers with preeclampsia, which acts as a neuroprotective agent in their neonates [7]. However, it is not obvious that whether preeclampsia itself has protective effect against IVH or not; and there is a small number of studies that considered the effect of pregnancy-induced hypertension and preeclampsia on IVH incidence in premature infants.

Thus, this study was designed to find any relationship between maternal preeclampsia and IVH in preterm infants weighting less than 1500 grams.

\section{Methods}

In this observational case-control study, all singleton newborns with a birth weight less than $1500 \mathrm{~g}$ born at Akbar-Abadi Hospital, Tehran, Iran during 2016-2017 entered the study. Demographic data of all newborns including gestational age, sex, and birth weight, as well as type of delivery, Apgar scores of 1 and 5 minutes were recorded. Brain ultrasonography was performed by an expert sonographist as screening evaluation by seventh day of life for all singleton premature newborns with a birth weight less than $1500 \mathrm{~g}$, andreports were recorded. Neonates with perinatal asphyxia, pneumothorax, major congenital anomalies, IUGR, trombocytopenia and coagulation disorders; and neonates died within the first 72 hours of life were excluded from the study. The history of hypertension prior to pregnancy or during early pregnancy and the presence of preeclampsia in mothers were recorded. Eventually, the relationship between previous hypertension with the occurrence of preeclampsia in mothers and the incidence of IVH in preterm neonates was evaluated.

Demographic and clinical characteristics of patients were reported as means \pm standard deviation for quantitative variables and as percentage for categorical qualitative ones. An independent student t-test was also considered to compare the mean of parametric data between IVH and non-IVH groups. Chisquare test was applied to compare the qualitative variables. Data were analyzed using SPSS software (version 19) and $p$ values less than 0.05 were assumed statistically meaningful. The research was approved primarily by ethics committee of Iran University of Medical Sciences with code number of IR.IUMS.REC.1397.1018. The objectives of the study were explained to the parents of all participants and written informed consent was obtained and they were clarified that data would be kept confidential and analyzed anonymously.

\section{Results}


A total of 305 preterm neonates enrolled the study. Demographic characteristics of all patients are shown in Table 1. Among 305 preterm newborn infants ( $51 \%$ boys and $49 \%$ girls), mean \pm SD gestational age was $29.26 \pm 2.53$ weeks, and mean \pm SD of neonates' birth weight was $1157.09 \pm 256.24 \mathrm{~g} .78 .6 \%$ of the preterm neonates were born by cesarean section method, while $21.4 \%(n=62)$ born by vaginal delivery. Apgar scores of 1 and 5 minutes were $6.46 \pm 1.97$ and $8.13 \pm 1.51$, respectively (Table 1 ).

Table 1

Clinical and demographic data of preterm neonates

\begin{tabular}{|ll|}
\hline Parameters & Results \\
\hline Sex & \\
\hline Boys & $153(51 \%)$ \\
\hline Girls & $147(49 \%)$ \\
\hline Gestational age (weeks) & $29.26 \pm 2.53$ \\
\hline Birth weight (g) & $1157.09 \pm 256.24$ \\
\hline Delivery type & \\
\hline Cesarean & $228(78.6 \%)$ \\
\hline Vaginal delivery & $62(21.4 \%)$ \\
\hline Apgar 1 & $6.64 \pm 1.97$ \\
\hline Apgar 5 & $8.13 \pm 1.51$ \\
\hline Preeclampsia & \\
\hline Yes & $56(18.4 \%)$ \\
\hline No & $249(81.6 \%)$ \\
\hline IVH & \\
\hline Yes & $82(26.9 \%)$ \\
\hline No & $223(73.1 \%)$ \\
\hline Hypertension & $265(86.9 \%)$ \\
\hline Yes & \\
\hline No & \\
\hline
\end{tabular}

In total, 46 (18.4\%) mothers had preeclampsia. Demographic and clinical data of neonates born from mothers with and without preeclampsia are presented in Table 2. The neonates of mothers with preeclampsia had significantly lower mean birth weight compared to the others $(1083.18 \pm 255.33 \mathrm{~g}$ vs 
$1173.48 \pm 254.05 \mathrm{~g} ; \mathrm{p}=0.018)$. None of mothers with preeclampsia had vaginal delivery $(\mathrm{p}<0.001)$. There were no significant difference in neonatal sex and gestational age and Apgar scores between neonates of mothers affected with preeclampsia and the others (Table 2).

Table 2

Clinical and demographic data of preterm neonates in mothers with and without preeclampsia

\begin{tabular}{|c|c|c|c|}
\hline \multirow[t]{2}{*}{ Parameters } & \multicolumn{2}{|l|}{ Preeclampsia } & \multirow[t]{2}{*}{ p-value } \\
\hline & Yes (56) & No (249) & \\
\hline Previous HTN & $16(40 \%)$ & $24(60 \%)$ & \multirow[t]{2}{*}{$<0.001$} \\
\hline No previous HTN & $40(15.09 \%)$ & $225(84.9 \%)$ & \\
\hline \multicolumn{4}{|l|}{ Sex } \\
\hline Boys & $21(39.62 \%)$ & 132 (53.44\%) & \multirow[t]{2}{*}{0.068} \\
\hline Girls & $32(60.37 \%)$ & $115(46.55 \%)$ & \\
\hline \multicolumn{4}{|l|}{ Delivery type } \\
\hline Cesarean & $55(23.4 \%)$ & $173(73.61 \%)$ & \multirow[t]{2}{*}{$<0.001$} \\
\hline Vaginal delivery & 0 & $62(100 \%)$ & \\
\hline Gestational age (Weeks) & $29.37 \pm 2.45$ & $29.24 \pm 2.56$ & 0.72 \\
\hline Birth weight (g) & $1083.18 \pm 255.33$ & $1173.48 \pm 254.05$ & 0.018 \\
\hline Apgar 1 & $6.47 \pm 1.78$ & $6.45 \pm 2.02$ & 0.94 \\
\hline Apgar 5 & $8.27 \pm 1.16$ & $8.1 \pm 1.57$ & 0.48 \\
\hline
\end{tabular}

Overall, 40 (13.1\%) out of 305 mothers had high blood pressure. Comparison of the demographic and basic clinical characteristics of neonates from mothers with and without hypertension is presented in Table 3. The hypertensive group had significantly higher mean Apgar score of 5 minute than the others ( $8.62 \pm 0.77 \mathrm{~g}$ vs $8.05 \pm 1.59 \mathrm{~g} ; \mathrm{p}=0.026$ ). Only $5 \%$ of hypertensive mothers underwent normal vaginal delivery, while $24 \%$ of normotensive mothers had vaginal delivery $(p=0.003)$. There was no significant difference in sex, gestational age, birth weight, and Apgar score of 1 minute between neonates of hypertensive and normotensive mother groups. 
Table 3

Comparison of the clinical and demographic characteristics between preterm neonates with and without IVH

\begin{tabular}{|c|c|c|c|}
\hline \multirow[t]{2}{*}{ Parameters } & \multicolumn{2}{|l|}{ IVH } & \multirow[t]{2}{*}{ p-value } \\
\hline & Yes $(n=82)$ & No $(n=223)$ & \\
\hline \multicolumn{4}{|l|}{ Sex } \\
\hline Boys & 43 (52.43\%) & $110(49.32 \%)$ & \multirow[t]{2}{*}{0.43} \\
\hline Girls & $39(47.56 \%)$ & $108(48.43 \%)$ & \\
\hline \multicolumn{4}{|l|}{ Delivery type } \\
\hline Cesarean & $61(78.2 \%)$ & 167 (78.77\%) & \multirow[t]{2}{*}{0.51} \\
\hline Vaginal delivery & $17(20.73 \%)$ & $45(21.22 \%)$ & \\
\hline Gestational age (Weeks) & $28.12 \pm 2.46$ & $29.69 \pm 2.43$ & $<0.001$ \\
\hline Birth weight (g) & $1011.64 \pm 245.38$ & $1211.06 \pm 239.06$ & $<0.001$ \\
\hline Apgar 1 & $5.81 \pm 2.11$ & $6.68 \pm 1.88$ & 0.001 \\
\hline Apgar 5 & $7.57 \pm 1.74$ & $8.33 \pm 1.37$ & $<0.001$ \\
\hline \multicolumn{4}{|l|}{ Preeclampsia } \\
\hline Yes & $9(16.07 \%)$ & $47(83.92 \%)$ & \multirow[t]{2}{*}{0.028} \\
\hline No & $73(29.31 \%)$ & $176(70.68 \%)$ & \\
\hline \multicolumn{4}{|l|}{ HTN } \\
\hline Previous HTN & $5(12.5 \%)$ & $35(87.5 \%)$ & \multirow[t]{2}{*}{0.018} \\
\hline No previous HTN & 77 (29.05\%) & 188 (70.94\%) & \\
\hline
\end{tabular}

The prevalence of intraventricular hemorrhage in preterm neonates was $26.9 \%(n=82)$. Comparison of the clinical and basic demographic characteristics between preterm neonates with IVH and without IVH is summarized in Table 3. There was no significant difference in the frequency of sex and deliver type between two groups. A significant difference was observed in the mean of gestational age, birth weight, Apgar 1 and 5, as well as the prevalence of preeclampsia between two groups. The mean gestational age of preterm neonates with IVH was significantly lower than that in non-IVH group $(28.12 \pm 2.46$ vs $29.69 \pm$ 2.43 weeks; $p<0.001)$. Preterm neonates with IVH had a lower mean of birth weight compared to non-IVH cases $(1011.64 \pm 245.38$ vs $1211.06 \pm 239.06 \mathrm{~g} ; \mathrm{p}<0.001)$. Non-IVH cases had significantly a higher mean Apgar scores at one and 5 minute $(6.68 \pm 1.88$ and $8.33 \pm 1.37$, respectively) compared to IVH group (5.81 \pm 2.11 and $7.57 \pm 1.74$, respectively) $(p<0.01)$. The incidence of IVH in preterm neonates of mothers with preeclampsia was $16.07 \%$, while it was estimated as $29.31 \%$ in preterm neonates of mothers without 
preeclampsia $(p=0.028)$. There was a significant difference in the prevalence of IVH in preterm neonates of mothers with a history of previous hypertension and without hypertension $(p=0.018)$. The prevalence of IVH in preterm neonates of mothers with hypertension and without hypertension was $12.5 \%$ and $29.05 \%$, respectively.

\section{Discussion}

In this study, we assessed the relationship between maternal hypertension and the incidence of IVH in preterm neonates weighting less than 1500 grams. Our data revealed that the prevalence of preeclampsia in mothers with hypertension was $40 \%$, while its prevalence in mothers without previous hypertension was $15.09 \%$. More recently, Gudeta et al., [12] have demonstrated that the prevalence of pregnancy induced hypertension was $7.9 \%$; of which $15.2 \%$ were gestational hypertension, $36.4 \%$ were mild preeclampsia, and $45.5 \%$ were severe preeclampsia. In another cohort study, Subki et al., [13] found that the incidence of preeclampsia in mothers with hypertension is $54.9 \%$. Therefore, these data indicate that pregnancy-induced hypertension is a major risk factor for the possibility of preeclampsia in pregnant mothers.

Our data also revealed that the overall incidence of IVH in preterm neonates was $26.88 \%$. Further analysis demonstrated that the incidence of IVH in preterm neonates of mothers with preeclampsia was $16.07 \%$, whereas it was accounted $29.31 \%$ in mother without preeclampsia. Nine $(10.97 \%)$ out of 82 neonates with IVH were born from mothers with preeclampsia. This data suggests that the incidence of neonatal IVH in mothers without preeclampsia is higher than that in mothers with preeclampsia; however, the underlying mechanism is not known. More recently, Morsing et al., [11] have demonstrated that maternal preeclampsia is associated with lower rates of IVH in preterm neonates ( $2 \% \mathrm{vs} 11 \%)$. We also found that the mean of birth weight in neonates of mothers with preeclampsia was significantly lower than that in mothers without preeclampsia (1083.18 g vs $1173.48 \mathrm{~g}$ ). Similarly, Morsing et al., [11] reported that preeclampsia group had a lower median birth weight than the background group $(795 \mathrm{~g}$ vs $890 \mathrm{~g}$ ). In another study, Mendola et al., [14] showed that the incidence of IVH in preterm infants of mothers with preeclampsia and normal blood pressure was $4.7 \%$ and $4.4 \%$, respectively. Paul et al., [7] didn't find any significant in IVH incidence and mortality rate between infants born to mothers with preeclampsia as compared to infants born to mothers without preeclampsia. It may be partly due to magnesium sulfate treatment in mothers with preeclampsia, which acts as a neuroprotective agent in their neonates $[7,15]$.

Our data have demonstrated that the prevalence of IVH in neonates of mothers without pregnancy hypertension is significantly lower than that in mothers with pregnancy hypertension. The incidence of IVH in preterm neonates of mothers with hypertension was $12.5 \%$, while its prevalence in mothers without hypertension was $29.05 \%$. Similarly, Perlman et al., [16] reported that the incidence of IVH in neonates of

mothers with pregnancy-induced hypertension is lower than that in mothers without hypertension (16\% vs $30 \%)$. A previous study revealed that IVH is less frequent in infants of mothers with preeclampsia compared with controls (4.8\% vs $20.5 \%$ ) [17]. Therefore, these findings indicate a significantly lower 
incidence of IVH of approximately $50 \%$ in preterm neonates from mothers with pregnancy-induced hypertension as compared with the incidence in infants born from mothers without hypertension.

We also found that IVH was significantly associated with decreased gestational age, birth weight, and Apgar scores. Preterm neonates with IVH had significantly lower gestational age, birth weight and Apgar scores compared to preterm neonates without IVH. Therefore, these parameters can be considered as a significant predicting factor for IVH. Some studies considered the gestational age, birth weight and Apgar scores as important risk factors for the prediction of IVH in preterm neonates. For example, Perlman et al., [16] identified that gestational age and birth weight are a significant predicting factor of IVH. Jodeiry et al., [18] reported that cases with IVH had significantly lower gestational age and Apgar scores than cases without IVH. They also found that vaginal delivery was significantly higher in cases with IVH, while in our study there was no significant difference in the frequency of vaginal delivery between two groups. In another study, Khalessi et al., [19] identified that gestational age, birth weight, mode of delivery, maternal hypertension and preeclampsia, pneumothorax, and ventilator therapy are important risk factors for the prediction of IVH in preterm neonates. In another study, Lee et al., [20] reported that metabolic acidosis and inotrope use increase the risk of IVH in neonates by 6.94 and 3.70 folds, respectively. Another study showed that lower gestational age and birth weight increases the risk of IVH by 3.72 and 3.42 times, respectively [21]. Therefore, according to accomplished studies and current research, pregnancy hypertension is significantly associated with preeclampsia, which can substantially increase the risk of preterm delivery.

\section{Conclusions}

Hypertensive disorders of pregnancy increases the risk of preterm delivery, but incidence of IVH in preterm neonates weighing less than $1500 \mathrm{gr}$ is lower in infants born to mothers with hypertension including preeclampsia compared to non hypertensive mothers. And also preterm neonates with IVH had significantly lower gestational age, birth weight and Apgar scores than preterm cases without IVH.

\section{Declarations}

\section{Ethics approval and consent to participate}

Ethics approval was obtained from the institutional review board of Iran University of Medical Sciences according to Helsinki declaration. The research was approved primarily by ethics committee of Iran University of Medical Sciences with code number of IR.IUMS.REC.1397.1018.

\section{Consent for publication}

Informed consent was written from all participants' parents before enrollment.

\section{Availability of data and materials}


The datasets used and analyzed during the current study are available from the corresponding author on reasonable request.

\section{Competing Interests}

The authors declare no conflict of interest.

\section{Funding}

The authors received no specific funding for this work.

\section{Author's contribution}

Mandana Kashaki , Maryam Saboute, and Marym Esmaeili Allafi gave substantial contribution to the conception or design of the work and in the acquisition, analysis and interpretation of data for the work. Mandana Kashaki, Marym Esmaeili Allafi and Elahe Norouzi had role in drafting the work and revising it critically for important intellectual content. Mandana Kashaki and Elahe Norouzi gave final approval of the version to be published and they agree to be accountable for all aspects of the work in ensuring that questions related to the accuracy or integrity of any part of the work are appropriately investigated and resolved. All authors have read and approved the manuscript.

\section{Acknowledgment}

The authors would like to express their gratitude to Shahid Akbarabadi Clinical Research Development Unit (ShACRDU), Iran University of Medical Scinces (IUMS), Tehran, Iran for their cooperation in this study.

\section{References}

1. Ballabh P. Intraventricular Hemorrhage in Premature Infants: Mechanism of Disease. Pediatr Res. 2010;67:1-8.

2. Whitelaw A. Intraventricular haemorrhage and posthaemorrhagic hydrocephalus: pathogenesis, prevention and future interventions. Semin Neonatol. 2001;6:135-46.

3. Szpecht D, Szymankiewicz M, Nowak M, Gadzinowski J. Intraventricular hemorrhage in neonates born before 32 weeks of gestation-retrospective analysis of risk factors. Childs Nerv Syst. 2016;32:1399-404

4. Wilson-Costello $D$, Friedman $H$, Minich N, Fanaroff $A A, M ~ H$. Improved survival rates with increased neurodevelopmental disability for extremely low birth weight infants in the 1990s. Pediatrics. 2005;115:997-1003.

5. Murphy BP, Inder TE, Rooks V, Taylor GA, Anderson NJ, Mogridge N, et al. Posthaemorrhagic ventricular dilatation in the premature infant: natural history and predictors of outcome. . Arch Dis Child Fetal Neonatal Ed. 2002;87:F37-F41. 
6. Pinto-Martin JA, Whitaker AH, Feldman JF, Van Rossem R, Paneth N. Relation of cranial ultrasound abnormalities in low-birthweight infants to motor or cognitive performance at ages 2, 6, and 9 years. . Dev Med Child Neurol. 1991;41:826-33.

7. Paul DA, Kepler J, Leef KH, Siscione A, Palmer C, Stefano JL. Effect of preeclampsia on mortality, intraventricular hemorrhage, and need for mechanical ventilation in very low-birth-weight infants. Am J Perinatol. 1998;15:381-6.

8. Volpe JJ. Intraventricular hemorrhage in the premature infant-current concepts. Part I. Ann Neurol. 1989;25:3-11.

9. Antoniuk S, da Silva RV. Periventricular and intraventricular hemorrhage in the premature infants. Rev Neurol. 2000;31:238-43.

10. Ballabh P, Braun A, Nedergaard M. Anatomic analysis of blood vessels in germinal matrix, cerebral cortex, and white matter in developing infants. Pediatr Res. 2004;56:117-24.

11. Morsing E, Maršál K, Ley D. Reduced Prevalence of Severe Intraventricular Hemorrhage in Very Preterm Infants Delivered after Maternal Preeclampsia. Neonatology 2018;114:205-11.

12. Gudeta TA, Regassa TM. Pregnancy Induced Hypertension and Associated Factors among Women Attending Delivery Service at Mizan-Tepi University Teaching Hospital, Tepi General Hospital and Gebretsadik Shawo Hospital, Southwest, Ethiopia. Ethiop J Health Sci. 2019;29:831-40.

13. Subki AH, Algethami MR, Baabdullah WM, Alnefaie MN, Alzanbagi MA, Alsolami RM, et al. Prevalence, Risk Factors, and Fetal and Maternal Outcomes of Hypertensive Disorders of Pregnancy: A Retrospective Study in Western Saudi Arabia. Oman Med J. 2018;33:409-15.

14. Mendola P, Mumford SL, Männistö TI, Holston A, Reddy UM, Laughon SK. Controlled Direct Effects of Preeclampsia on Neonatal Health After Accounting for Mediation by Preterm Birth. Epidemiology. 2015;26:17-26.

15. Ehsanipour F, Norouzi E, Khosravi N, Mirfakhraee H. Serum Magnesium Levels in Neonatal Jaundice: Is There any Correlation Between Hypermagnesemia and Moderate to Severe Hyperbilirubinemia in Term Newborns? J Compr Ped. 2020 May;11(2):e94278

16. Perlman JM, Risser RC, Gee JB. Pregnancy-induced hypertension and reduced intraventricular hemorrhage in preterm infants. Pediatr Neurol. 1997;17:29-33.

17. Shah DM, Shenai JP, Vaughn WK. Neonatal outcome of premature infants of mothers with preeclampsia. J Perinatol. 1995;15:264-7.

18. Jodeiry B, Heidarzadeh M, Sahmani-Asl S, Hoseini M, Javaherizadeh H, Eliasi S, et al. Study of intraventricular hemorrhage in VLBW neonates admitted in Al-Zahra Hospital, Tabriz, Iran. Niger J Med. 2012;21:92-7.

19. Khalessi N, Farahani Z, Shariat M, Rezaeizadeh G. Risk factors of intracranial hemorrhage in premature neonates. Acta Med Iran. 2014;52:687-9.

20. Lee JY, Kim HS, Jung E, Shim GH, Lee HJ, Lee JA, et al. Risk Factors for PeriventricularIntraventricular Hemorrhage in Premature Infants. J Korean Med Sci. 2010;25:418-24. 
21. Khodapanahandeh F, Khosravi N, Larijani T. Risk factors for intraventricular hemorrhage in very low birth weight infants in Tehran, Iran. Turk J Pediatr. 2008;50:247-52. 